\title{
Towards a pragmatic modeling of learner's complex system by reflecting Boulding's typology at the affective computing space
}

\author{
Sofia J. Hadjileontiadou ${ }^{1}$, Georgia N. Nikolaidou ${ }^{2}$, Leontios J. Hadjileontiadis ${ }^{3}$ \\ 1. Division of Environmental Education, Hellenic Open University, Greece. 2. Division of Primary School of Education, \\ Prefecture of Eastern Thessaloniki, Greece. 3. Department of Electrical \& Computer Engineering, Aristotle University of \\ Thessaloniki, Greece.
}

Correspondence: Leontios J. Hadjileontiadis. Address: Department of Electrical \& Computer Engineering, Aristotle University of Thessaloniki, University Campus, Build. D-6th floor, Thessaloniki 546 45, Greece. E-mail: leontios@auth.gr.

Received: July 16, 2012

DOI : $10.5430 /$ air.v2n1p36

Accepted: September 23, 2012

URL: http://dx.doi.org/10.5430/air.v2n1p36
Online Published: November 27, 2012

\section{Abstract}

This work reflects Boulding's Typology (BT) of the learner's complex system at the space of affective computing. From this perspective, the learner's emotional state is interweaved with the structural elements of his/her learning functioning (both internal and external) when placed within an educational setting. The advent of new technological achievements in the accurate acquisition of the learner's affective state allows for the redesign of the ICT-based educational settings, taking into account a bilateral approach of the learner's system that involves both cognitive and emotional processes, as reflected in the Valence/Arousal space. Justification of the crucial role of learner's affective state in the design of an ICT-based educational setting is provided with the implications derived from an experimental case-study, referring to emotional responses to IADS-based sound stimuli presented to three age-dependent learner-groups. The proposed affective parameters define an enriched BT that could serve as a basis for structuring a new model, closer to the pragmatic nature of the learner's system.

\section{Key words}

Boulding's typology, Affective computing, Valence, Arousal, Sound stimuli emotional responses

\section{Introduction}

Many times within the educational setting, statements like "I can't do this" and "I'm not good at this" are common during the learning effort. In most of cases, these thoughts are not being addressed, despite the importance of their source, i.e., learners' affective states of confusion, frustration, and hopelessness. The polished form of the educational material presented in the class by the teachers omits the natural steps of making mistakes (feeling confused), recovering from them (overcoming frustration), deconstructing what went wrong (not becoming dispirited), and starting over again (with hope and maybe even enthusiasm) ${ }^{[9]}$. Nevertheless, learning naturally involves failure and becomes a host of associated affective responses. 
Emotional intelligence, that is the ability to identify, assess, and manage emotions of one's self and of others, plays a crucial role in learning processes and, particularly, in the capability of extracting the information that is most important ${ }^{[6]}$. Furthermore, a number of studies by neuroscientists, cognitive scientists and psychologists have shown that emotion is of major importance in rational and intelligent thinking.

Within the aforementioned perspective, there is a clear necessity to view learning as a person's ability to construct new knowledge based upon what s/he already knows or believes to be true ${ }^{[4]}$, by performing model-based reasoning, recursion, and cognitive assessment, i.e., metacognition, without neglecting, though, the influence of emerged affective states. The latter could then lead the system of external representation of knowledge (e.g., teacher and/or ICT-based educational supporting system) to respond in an appropriate-adaptive manner (e.g., modulate the pace, alter and/or redefine the direction or complexity of the presentation). Towards such direction, Kort et al. ${ }^{[9]}$ have tried to form a model that describes the range of various emotional states during learning. In particular, they defined a xyz-space, where the x-axis corresponds to an Emotion Axis, symbolizing an n-vector of all relevant emotion axes, thus, allowing multi-dimensional combinations of emotions. For example, the x-axis could represent (from negative valence, i.e., more unpleasant, emotions to positive valence, i.e., more pleasant, emotions): \{Anxiety - Confidence: anxiety - worry - discomfort - comfort - hopeful confident\}, \{Boredom - Fascination: ennui - boredom - indifference - interest - curiosity - intrigue\}, \{Frustration Euphoria: frustration - puzzled - confusion - insight - enlightened - epiphany\}, \{Dispirited - Encouraged: dispirited disappointed - dissatisfied - satisfied - thrilled - enthusiastic $\}$, \{Terror - Enchanted: Terror - Dread- Apprehension - Calm - Anticipatory - Excited\}. The y-axis refers to the Learning Axis, symbolizing the construction of knowledge upward, and the discarding of misconceptions downward. In addition, the z-axis denotes the Knowledge Axis, adopting an excelsior spiral when evolving/developing knowledge. In building a complete and correct mental model associated with a learning opportunity, the learner may experience multiple cycles around the xyz-plane until completion of the learning exercise. In this trajectory, each orbit represents the time evolution of the learning cycle, gradually moving up the knowledge z-axis.

Kort et al.'s model ${ }^{[9]}$ focuses on the range of emotional states during learning, trying to somehow decode, from a cause-effect distant view, their role in the learning process. In this paper, however, an alternative approach is undertaken. In particular, an effort to shed light upon the way affective states emerge within the learning process is initiated, placing the focus at their effect in the internal structure of the learner's system. To achieve this, $\mathrm{BT}^{[1]}$ that describes a system's complexity is adopted as a model-basis; the latter is then appropriately enriched with affective state parameters, providing an integrated model that could contribute to a more efficient knowledge-representation organization and handling. Implications from emotional responses in sound stimuli within three age-dependent educational settings are also provided, as a case-study of the way affective parameters should be taken under consideration in the design of ICT-based educational settings.

\section{The proposed approach}

\subsection{Description of the BT}

BT aims at defining a system's components by uncovering the "hard" facts (e.g., laws in the "hard" natural sciences) of "soft" social systems (e.g., learning, collaborative settings) ${ }^{[1]}$. According to this typology, the systems of the world are ranked from simple to complex in nine levels as follows: 1) Frameworks (systems with static structures), 2) Clockworks (simple dynamic systems with predetermined motions), 3) Thermostats (cybernetic systems, i.e., self-regulated on the basis of an external prescribed criterion), 4) Open systems (systems that are self-maintained on the basis of resources from the environment, e.g., the cell), 5) Blue-printed growth systems (systems that contain pre-programmed instructions for development, e.g., the plant), 6) Internal-image systems (systems capable of a detailed awareness of the environment through sense organs, e.g. the animal), 7) Symbol-processing systems (systems that use language and other symbols, are self-conscious, and can contemplate the past, present, and future e.g., humans), 8) Social systems (comprising actors from 
level 7 who share a common social order and culture, e.g., social organizations), and 9) Transcendental systems (systems composed of the "absolutes and the inescapable unknowables").

In the above typology, levels 1-3 comprise the things of the system and are designable, i.e., externally regulated to externally prescribed criteria, (levels 1 and 2) and self-regulated to externally prescribed or designable criteria (level 3). The levels 4-7 comprise the people, thus, they are undesignable, i.e., self-regulated to internally prescribed criteria (levels 4 -7). Finally, levels $8-9$ represent the social outcomes, which are also undesignable, since they are intangible ${ }^{[5]}$. This distinction clarifies which levels (subsystems) are predictable and controllable within a system and which are not, and classifies the relevant types of external (inputs) or internal (attractors) stimuli for their function. Moreover, since each level in this hierarchy incorporates all below ${ }^{[1]}$, it is easily realized that when predictable and designable inputs are designed at levels 1-3, they are expected first to be perceived at level 6 and then reformed to attractors of intrinsic motivation at level 7, towards fulfilling initially individual and then social goals from level 8 and up. Figure 1 depicts the levels involved in the BT.

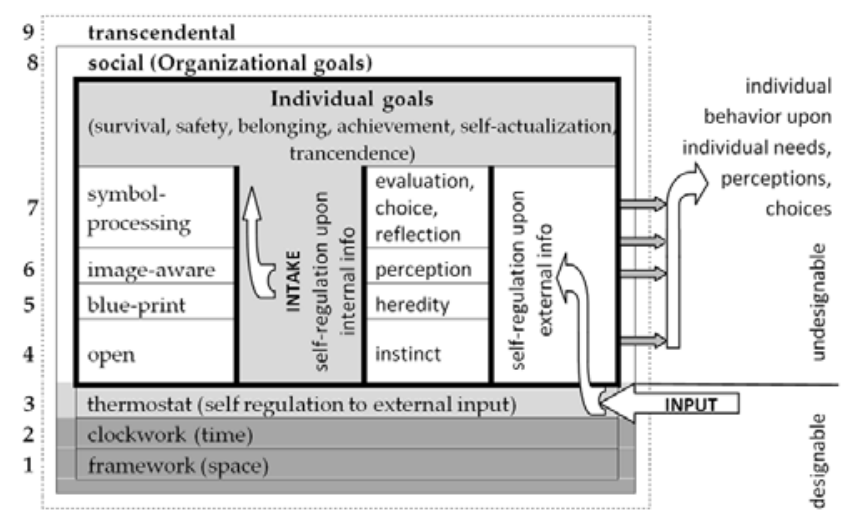

Figure 1. The level-based structure of BT.

Transferring BT to the educational space, examples of the first two levels in schools are formal goals, participant roles, equipment and rooms for frameworks, calendars, schedules and routine activities for clockworks. Examples of the third, i.e., the level with the thermostat metaphor, are evaluations of the recourses and needs and estimation of the workload in the classroom that leads to self-regulated remedy actions, if needed. The first three levels that comprise the things of the system, are either externally (levels 1 and 2) or self-regulated (level 3) to externally prescribed inputs. In level 4, e.g., educational resources, information, programs should be available to the individual who is acting according to interior criterion (instinct), thus, the input here is described as 'intake' ${ }^{[5]}$. Level 5 realizes the variability of the interior criterion due to the differences of the people (heredity), which is inherited to the response to the stimulus. Furthermore, in level 6, the individual perception intervenes between the stimulus and the response; hence, from this level on, the input/intake is described as 'pickup' ${ }^{[5]}$. The individual 'picks up' whatever she/he believes in or understands, otherwise his/her output will be distorted by his/her perception, e.g., instead of learning, memorizing. At level 7, the individual acts consciously to produce the output according to his/her perceptions of the 'pickup', but even upon new stimulus that she/he can create from the processing of his/her past experiences. The 'people' levels are characterized by physical boundaries. Yet, at levels 8 and 9 , where the social outcome is expected, only if the individual participation is voluntary and authentic the social intangible system will exist. Moreover, at these levels, individual goals are to be met before the social, otherwise the individual seeks his/her own ${ }^{[5]}$.

\subsection{Lerner's affective states modeling and recognition}

Psychologists do not present emotions as discrete states, but rather as continuous ones and therefore demonstrate them in an n-dimensional (n-D) space; usually the 2-D Valence/Arousal Space (VAS) is adopted. Valence stands for one's 
judgment about a situation as positive or negative and arousal spans from calmness to excitement, expressing the degree of one's excitation. This approach stems from Lang's perspective ${ }^{[11]}$ that two motive systems exist in the brain, i.e., appetitive and aversive, accounting for the primacy of the valence dimension. As far as the arousal dimension is concerned, this is not viewed as having a separate substance, but rather, as reflecting variations in the activation of metabolic or neural or both systems. Tactical demands of context may variously shape affects, which are, however, organized around a motivation base. In this vein, valence and system arousal comprise the strategic dimensions of the emotion world. Figure 2 presents an example of the VAS, consisting of the four quadrants, i.e., High Valence-High Arousal (HVHA), Low Valence-High Arousal (LVHA), Low Valence-Low Arousal (LVLA) and High Valence-Low Arousal (HVLA).

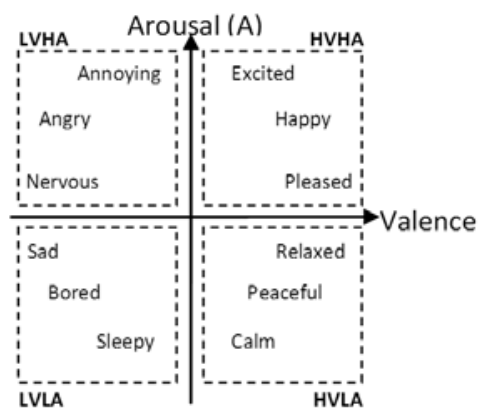

Figure 2. The VAS with the four quadrants, i.e., HVHA, LVHA, LVLA, HVLA, including some basic emotion examples.

The manual approach of emotion recognition is observation by skilled humans or self-assessment through emotion reporting forms. The latter most often refers to the Self-Assessment Manikin (SAM) form ${ }^{[2]}$ that depicts the V/A dimension with a graphical character arrayed along a continuous nine-point scale. For valence, SAM ranges from smiling, happy figure to frowning, unhappy figure; for arousal, SAM ranges from sleepy with eyes closed to excited with eyes open (Note 1) (see Figure 3). Apart from the manual emotion recognition approaches, significant research effort has been placed, nowadays, towards effective recognition of emotions from various human sources. Some examples include: face expression data, voice data, and data related to the autonomous nervous system (galvanic skin response, heart rate, etc) ${ }^{[14]}$. Currently, new efforts have been initiated to recognize emotions with electroencephalogram (EEG)-based recognition systems, by artificially eliciting emotional states (Petrantonakis and Hadjileontiadis, $2010^{[13]}$ ). The most frequently used technique to evoke emotion during an emotion recognition procedure is to use pictures or sounds related to situations or events that are supposed to elicit affective states relying in the VAS. International Affective Picture System (IAPS) ${ }^{[12]}$ and International Affective Digitized Sound (IADS) ${ }^{[3]}$ are two widely used datasets, containing such pictures/sounds, which come with their individual values of valence and arousal.

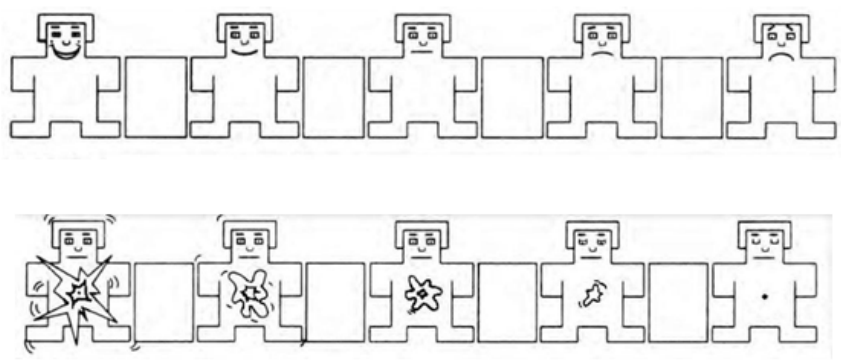

Figure 3. The valence (top) and arousal (bottom) range representation via the SAM graphic characters.

Despite the potential of the classification of the aforementioned methods, further research is needed in order to improve recognition rates and discover unknown aspects of emotion mechanisms performed in the human body and behavior. From 
a practical point of view, though, relevant flexible, user-friendly and wireless devices have recently came into foreground; such an example is EPOC EMOTIV (www.emotiv.com), which captures the user's EEG data and, by analyzing them, returns his/her emotional state. Such technological achievements could contribute to the accurate identification of a learner's cognitive-emotive state, setting a critical indicator that shapes the design of an ICT-based supporting educational system towards a more pragmatic functioning, and determines the way the learner could be assisted in appreciating an understanding of the efficiency and pleasure of the learning process.

\subsection{Enrichment of BT with affective parameters}

In the light of the affective states modeling using the VAS, the described BT could be enriched with affective parameters, forming a model that could incorporate the effect of the learner's current affective state to structural components of his/her learning system. As it was shown in Section 2.1, BT's levels 4-7 are undesignable, i.e., self-regulated to internally prescribed criteria. These are, in fact, the main levels where affective states could provoke their highest impact, according to the V/A affective status. In particular, the learner's instincts that set the system goals at level 4 could be fostered or hampered by the activation of the corresponding VAS quadrant. To this end, the learner's triggering (Trig) of using the available intakes is parallelized to a transition between VAS quadrants, converging to the HVHA one, i.e.:

$$
\operatorname{Trig}^{L_{4}}=I_{C} *\left\{\left.V A\right|_{V A \rightarrow H V H A}\right\} / I_{\max }
$$

where $I_{c}$ and $I_{\max }$ denote the current and maximum power of the learner's instinct, respectively, whereas $V A$ corresponds to the current affective state, expressed through the VAS level.

At the next level 5, the interior criterion increases in complexity and variability, producing more complex patterns of heredity. In such case, the affective state could evoke a multitude of internally script responses, thus increasing the power of heredity (Her), and adding to the latter through the multitude $k$ of the evoked emotions that form the total affective state itself, i.e.:

$$
\operatorname{Her}^{L_{5}}=\sum_{i} R_{i} *\left(V A_{i}\right)^{k}
$$

where $R_{i}$ denote the ith internal response and $V A_{i}$ corresponds to the current VAS level of affective state.

At level 6, an image or perception intervenes between the stimulus (St) and the response (Rsp). Consequently, the effect of affective state could be on the sign of this learner's image of stimulus (Image (St)), taking into consideration the valence $\operatorname{sign}(<5 \rightarrow-,>5 \rightarrow+)$ only at the VAS, i.e.:

$$
\operatorname{Rsp}^{\mathrm{L}_{6}}=\operatorname{Image}(\mathrm{St})^{*} \operatorname{sign}\left(\left.\mathrm{VA}\right|_{\mathrm{V}}\right)
$$

Finally, at level 7, the response/output/outcome (Rsp) depends on the choice of the individual who not only choose what $\mathrm{s} /$ he perceives and needs and ignores other stimuli (pickup), but $\mathrm{s} / \mathrm{he}$ can create new stimulus from processing and contemplation of past experience or beliefs. The affective state could affect the forming of these past experiences and beliefs weighting them with an affective mood parameter (Mood) created by the contribution of all affective states (previous and current), affecting the learner's current stimulus initiatives $\left(S t_{\text {new }}^{L 7}\right)$, i.e.:

$$
R s p^{L_{7}}=\left[\mathrm{F}\left(R s p^{L_{6}}, S t\right)+S t_{\text {new }}^{L_{7}}\right] * \operatorname{Mood}\left(\sum_{i} V A_{i}\right)
$$

where $\mathrm{F}$ denotes the blending function of past responses $\left(R s p^{L 6}\right)$ and stimuli $(S t)$.

As a far as the rest of the BT levels is concerned, the affective state could influence them too, mostly levels 3,8 and 9. At level 3, the system (e.g., classroom) self-regulates to an externally prescribed criterion or goal through an ongoing bimodal adjustment, "on/off", like the functioning of a thermostat (T1). In this metaphor, the thermostat (e.g., the teacher) could 
adjust the workload according to student needs, course description, and environment in the classroom social context. Let us imagine that a second thermostat (T2) exists that measures the social affective state of the learners, as the equilibrium achieved by the integration of their affective state within a time window. For example, the teacher expresses an unfair judgment to a student; the emotion of anger would probably dominate amongst the students. In such a case, the T2 will dominate to the functioning of $\mathrm{T} 1$ and the learning process would be saturated not due to the work overload but due to the negative emotional overwhelming of the learners. In an opposite scenario, where a positive emotion is conveyed amongst the students, $\mathrm{T} 2$ could facilitate the functioning of $\mathrm{T} 1$ and it could potentially increase its tolerance, as the students, due to their positive affective state, could bare more work load, than usually do. At levels 8 and 9 , the social outcome is expected. This means that the externalization of the learner's educational goals and purposes are expressed through his/her social interactions within the social setting (group, classroom, society) that clearly are affected by his/her affective state. The latter could, eventually, control a smooth or rough transition towards self-actualization and transcendence (the highest goal in education). Overall, the embodiment of affective parameters in the BT offers an extended opportunity to better understand the learner as a functioning system. This is examined, in part, in the experimental case-study that follows.

\section{Experimental case-study}

\subsection{Rationale \& experimental setup}

The rationale behind this experimental study was the testing of the way VAS changes, when learners from three different age-dependent educational settings are subjected to the acoustic stimuli of IADS. Implications from this case-study could add to role of affective perception of a stimulus (here the sound) activated in BT's level 4, which, due to its abstract form, could evoke spontaneous emotions and trigger the activation of the next levels of the enriched BT, presented in Section 2.3 .

Table 1. Type and order of IADS stimuli of the case-study

\begin{tabular}{|c|c|c|}
\hline Stimuli ID & Stimuli Name & VAS Quadrant \\
\hline 353 & Baseball & HVHA \\
\hline 275 & Scream & LVHA \\
\hline 723 & Radio & LVLA \\
\hline 809 & Harp & HVLA \\
\hline 810 & Beethoven & HVLA \\
\hline 367 & Casino2 & HVHA \\
\hline 286 & Victim & LVHA \\
\hline 424 & CarWreck & LVHA \\
\hline 812 & Choir & HVLA \\
\hline 700 & Toilet & LVLA \\
\hline 352 & SportsCrowd & HVHA \\
\hline 151 & Robin (bird) & HVLA \\
\hline 708 & Clock & LVLA \\
\hline 817 & Bongos & HVHA \\
\hline 279 & Attack1 & LVHA \\
\hline 728 & Paper1 & LVLA \\
\hline
\end{tabular}


The experiment was conducted on 71 pupils (30 boys, 41 girls), 8-12 yrs old; 11 university students ( 8 men, 3 women), 21-26 yrs old, and 15 teachers (4 men, 11 women), 34-50 yrs old. The experimental setup included 16 sounds from the IADS database (see Table 1), covering the whole VAS. The sounds were appropriately selected to comply with the subjects' age range of 8-50 yrs. The sounds were presented to the subjects in a pseudorandom order, not known prior to them, as a means to reduce predictably and increase the emotion variability; hence, the independence of the emotional responses. The experiment lasted $\sim 15$ mins; each presented sound lasted $6 \mathrm{~s}$, followed by a $30 \mathrm{~s}$ silence, where the subjects were asked to self-report their V/A levels via the SAM form (see Figure 3). This silent period also acted as a means of emotional neutralization. Three seconds before the initiation of each stimulus, a neutral beep sound was presented, for preparation and concentration. Data analysis was carried out using Matlab 2011 (The Mathworks, Natick, NJ).

\subsection{Results \& discussion}

An indicative sample of the whole experimental results set is presented here, as a means to foster the dependence of the affective perception of the acoustic stimuli to the age differentiation. In particular, Figure 4 depicts the mean V/A values per age-group (pupils, students, teachers), considering, though, as a VAS stimuli norm basis not the one from IADS, but the categorization performed by the teachers. This provides a means to measure the deviations from the teachers' perspective in the VAS responses of the other two age-groups. From Fig. 4 it could deduced that teachers did not activated all quadrants of the VAS, focusing mainly on LVHA and HVHA. This fact denotes a bias in their affective response, presenting a high deviation in $\mathrm{V}$ values and high A values. On the other hand, students and, especially pupils, exhibited a higher "affective response resolution", since they activated all VAS quadrants, complying with the character of the stimuli.

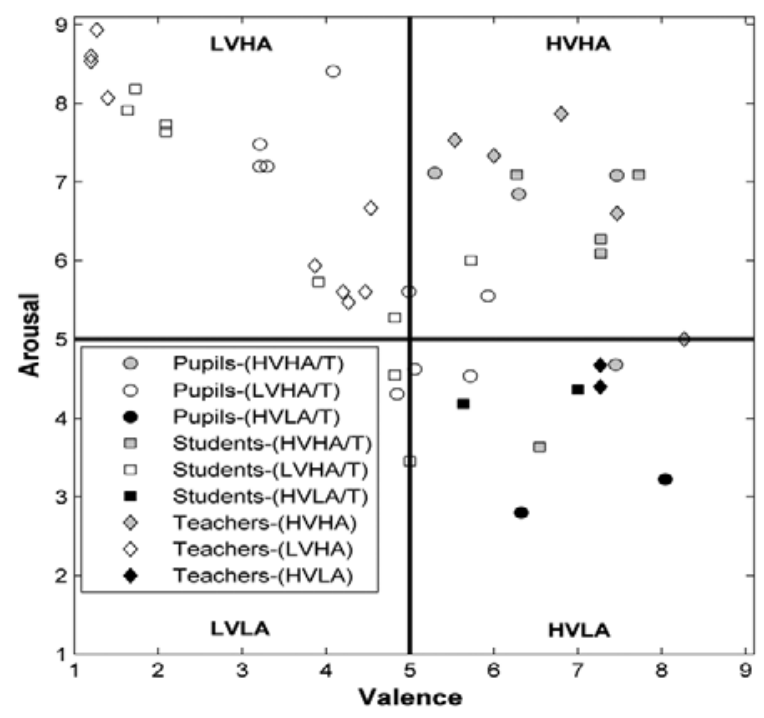

Figure 4. Mean V/A values per age-group. $T$ in legend symbolizes that the Teachers' categorization was used a stimuli norm basis.

The above findings concur with the findings of Grühn and Scheibe ${ }^{[7]}$, who, based on IAPS stimuli, they also found a bias towards the extremes of the LVHA, as the age increases. A possible explanation of this affective behavior is the dedifferentiation of the emotional space with age. According to ${ }^{[10]}$, the emotional understanding changes with age. In fact, it increases during the transition from the adolescence to middle age and after a plateau starts to decrease, as the age increases. This example of age-dependent affective behavior could be reflected back to the enriched BT-based model of the learner's system (Section 2.3), as an example of the way the affective parameters, expressed in (1)-(4), could affect the learning process. For example, in a music education setting, where sound perception is the learning goal itself, the aforementioned difference in the affective perception of the sound stimuli with age would affect the way enriched BT levels would be activated. Consequently, it is clear that such behavior of the learner's system should be used to guide the 
design of an integrated ICT-based educational setting that takes into account cognitive and emotional state of the learner; authors' related work ${ }^{[8]}$ paves the way towards such direction.

\section{Conclusions}

In this work, an insight within the learner's system was attempted, by employing Boulding's typology enriched with affective state parameters. This approach allowed for interweaving the learner's emotional state within the structural elements of his/her learning functioning (internal and external) within an educational setting. The implications derived from an experimental case-study referring to emotional responses to sound stimuli support the proposed approach, justifying the crucial role of learner's affective state in the design of an ICT-based educational setting.

\section{Acknowledgments}

The authors would like to thank all 97 participants who voluntarily contributed to the realization of the experimental case-study.

\section{References}

[1] K E Boulding. General systems theory-the skeleton of science. Management Science 1956; 2(3): 197-208. http://dx.doi.org/10.1287/mnsc.2.3.197

[2] M M Bradley, P J Lang. Measuring emotion: The self-assessment manikin and the semantic differential. J of Behavioral Therapy \& Exp. Psychiatry. 1994; 25(1): 49-59. http://dx.doi.org/10.1016/0005-7916(94)90063-9

[3] M M Bradley, P J Lang. The International Affective Digitized Sounds (2nd Edition; IADS-2): Affective ratings of sounds and instruction manual. Technical report B-3, Univ. of Florida, Gainesville, FL; 2007.

[4] B Cobb. Theories of mathematical learning and constructivism: a personal view. Proc. Symp. on Trends and Perspectives in Mathematics Education, Institute for Mathematics, University of Klagenfurt, Austria; 1994

[5] S Gabriele. Boulding's typology elaborated: A framework for understanding school and classroom systems. Systems Practice. 1997; 10(3): 271-303. http://dx.doi.org/10.1007/BF02557899

[6] D Goleman. Emotional Intelligence. New York, NY: Bantam Books; 1995.

[7] D Grühn S Scheibe. Age-related differences in valence and arousal ratings of pictures from the International Affective Picture System (IAPS): Do ratings become more extreme with age? Behavior Research Methods. 2008; 40(2): 512-521. http://dx.doi.org/10.3758/BRM.40.2.512

[8] S J Hadjileontiadou, G N Nikolaidou, L J Hadjileontiadis. An intuitionistic fuzzy logic-based approach of intrinsic motivation in CSCL settings during illusionary sense of control. In A. Peña-Ayala (Ed.), Intelligent and adaptive educational-learning systems: Achievements and Trends, KES-Springer Verlang Book Series: Smart innovation, systems and technologies (Chapter 18); 2013.

[9] B Kort, R Reilly, R W Picard. External representation of learning process and domain knowledge: Affective state as a determinate of its structure and function. Proc. Artificial Intelligence in Education (AI-ED 01) [Internet]. 2001. Available from: http://affect.media.mit.edu/projectpages/ lc/AI-ED.html

[10] G Labouvie-Vief, M G Marquez. Dynamic integration: Affect optimization and differentiation in development. In D. Y. Dai \& R. J. Sternberg (Eds.), Motivation, emotion, and cognition: Integrative perspectives on intellectual functioning and development, Mahwah, NJ: Erlbaum. 2004: 237-272.

[11] P J Lang. The emotion probe: studies of motivation and attention. American Psychologist. 1995; 50(5): 372-385. PMid:7762889 http://dx.doi.org/10.1037/0003-066X.50.5.372

[12] P J Lang, M M Bradley, M. M., B N Cuthbert. International Affective Picture System (IAPS): affective ratings of pictures and instruction manual. Tech. Rep. A-8, Univ. of Florida, Gainesville, FL; 2008.

[13] P C Petrantonakis, L J Hadjileontiadis. Emotion recognition from EEG using higher order crossings. IEEE Trans. on Inf. Tech. in Biomed. 2010; 14(2): 186-197.

[14] R W Picard, E Vyzas, J Healey. Toward machine emotional intelligence: analysis of affective physiological state. IEEE Trans. on Pat. Anal. and Mach. Intel. 2001; 23: 1175-1191. http://dx.doi.org/10.1109/34.954607 